\title{
Real-time evolution of oil shale meso-structure under triaxial stress condition
}

\author{
Haibo Tang ${ }^{1 *}$, Zhiqin $\mathrm{Kang}^{2}$, Kun Wang ${ }^{3}$ \\ ${ }^{1}$ College of Mining Engineering, Taiyuan University of Technology, 030024 Taiyuan Shanxi China \\ ${ }^{2}$ Mining Institute of Technology, Taiyuan University of Technology, 030024 Taiyuan Shanxi China \\ ${ }^{3}$ Shanxi Xinxin Composite Technology Co., Ltd, 030100 Taiyuan Shanxi China
}

\begin{abstract}
It is significant to research the mechanical properties and micro-fracture evolution characteristics of oil shales with different bedding directions under the condition of in-situ triaxial stress loading for understanding the internal micro-characteristics of oil shales. Triaxial stress loading tests of cylindrical oil shales were carried out in two loading directions perpendicular to and parallel to the bedding, which is the S1 and S2 samples. Our results demonstrate that the oil shales are typical anisotropic and brittle fracture characteristics. The triaxial compressive strength of S1 is higher than that of S2, but the elastic modulus is almost the same. Based on the reconstructed 3D-CT image, we analyzed the dynamic evolution law of the fracture inside the sample in the horizontal and vertical directions, and obtained the distribution of the fracture in the loading process, finally revealing the internal micro-characteristics of oil shales under the different loading conditions.
\end{abstract}

\section{Introduction}

With the booming economic development, the demand for energy continues to rise. The long-term high-intensity exploitation of coal resources in China and the continuous increase of oil dependence on foreign countries make the development and utilization of oil shale resources gradually come into people's vision[1,2]. Oil shale, a kind of unconventional energy and rich in organic matter, has the potential to be a major energy resource in the future due to its typical characteristics of high reserves and wide distribution. China has abundant oil shale reserves, about 2 trillion tons, of which the proven reserves are up to 31.57 billion tons, mainly distributed in Fushun, Maoming, Huadian and Xinjiang.

In the process of oil shale in-situ distillation, drilling input wells, production wells and pre-fracturing rock strata are important steps for accomplishing industrial production. However, the deformation and failure characteristics of rock mass during construction will directly affect the stability of wellbore and surrounding rock. Based on the characteristics of shale bedding development and anisotropy, it is of great significance to study the macroscopic mechanical properties and deformation and failure of oil shale to promote the safe exploitation of resources. In 1967, Tisot evaluated the changes in the compressive strength, structure, gas permeability, porosity, density and other physical properties of the Luhe oil shale with different organic matter contents under different heating temperatures[3]. In 1983, duvall studied the variations of thermal expansion and structural damage of different grades of
Green river oil shale under different temperatures and loads[4]. In 2018, Burnham deduced an anisotropic thermodynamic model of oil shale deformation in Piceance basin based on a large number of field and laboratory data[5]. In 2016, White proposed a thermoplastic model that can be applied to thermodynamic experiments, which can relate macroscopic strength to microstructure evolution through simple composition models[6]. In 2013, Kang tested the permeability coefficient of Fushun oil shales under different temperatures and triaxial stresses[7]. In 2020, Wang and Yang made a detailed report on the properties of Fushun oil shales in different bedding directions according to the anisotropic properties of oil shales[8,9]. In addition, according to the principle of MTI technology, Yang simulated the changes of temperature field, stress field and flow field in the pyrolysis process of oil shale rock under the THMC coupling condition in 2019[10]. Fan, Lee simulated the underground in-situ evolution process of oil shale based on Shell's ICP technology[11,12].

It is well known that the macroscopic deformation properties of rocks under external conditions start from the small changes in the internal microscopic structure. However, most of studies on oil shale do not synchronously involve to the internal meso-structure mechanism of rock, and just observe the failure process macroscopically. In other research fields, in order to timely and intuitively capture the phenomenon of internal damage and failure of rock materials under external loading conditions, people have begun to pay attention to the combination of observation equipment and

*Corresponding author: Haibo Tang, Email: tanghaibo0073@link.tyut.edu.cn 
experimental equipment. The computed tomography technology has been widely used in geological research as an intuitive non-damage scanning technique. In 2018, Ju recorded the evolution of internal fracture network of coal samples under different loads by servo-controlled triaxial loading and computer tomography, and quantitatively characterized it with fractal method[13]. In 2019, Zhang used X-ray microscopic tomography to carry out real-time imaging of the water-bearing heterogeneous coal before and after supercritical $\mathrm{CO} 2$ injection, and found that the partial dissolution of the calcite-pyrite composition significantly improved the porosity and connectivity of the sample[14]. In 2019, saif used time-resolution X-ray scanning technology to analyse the rules of initiation, expansion, connectivity and closure of pore and fracture in the pyrolysis process of oil shales and to obtain the evolution characteristics of the fractures[15]. In this study, we conducted simultaneous experimental observation on the macroscopic mechanical properties and internal structure evolution characteristics of oil shales samples based on micro-focus CT scanning technology, and then recorded the deformation of oil shales samples at different loading stages, and characterized the development and evolution of internal fracture morphology.

\section{Oil shale sample and experimental procedure}

\subsection{Sample preparation}

The oil shales samples in this study were obtained from Fushun, Liaoning, China, which is one of the main oil shales reservoirs in China. Complete oil shales samples were taken on site and immediately sealed with thin film to prevent oxidatio. A number of $5 \mathrm{~mm}$ diameter and $10 \mathrm{~mm}$ high cylinders were prepared from irregular oil shales blocks by using a small drilling machine. The cylinders were divided into vertical and parallel bedding directions. After fine grinding and polishing, the cylinder samples were finally obtained. In this test of mechanical properties, S1 sample is perpendicular to the bedding and S2 sample is parallel to the bedding.

\subsection{Experimental apparatus}

In this study, we have adopted the CT-THMC coupling test system developed by Professor Zhao Yangsheng from Taiyuan University of Technology. The system consists of a high precision micro-CT system and a THMC coupling test system. The micro-CT system can scan all kinds of materials and accomplish 3D reconstruction, it's up to 400 times at magnification and can achieve minimum $0.5 \mu \mathrm{m}$ pixel recognition. The diameter of specimen can be scanned is as large as $50 \mathrm{~mm}$ and as small as $1 \mathrm{~mm}$, see Fig.1. The bearing turntable adopts high precision servo control movement to realize precise positioning. The THMC coupling testing equipment is placed on the turntable to realize real-time scanning during loading, see Fig. 2 .
After the oil shale sample is installed, when the conical beam passes through the sample, the array detector captures the transmitted X-ray beam and converts it into digital image according to the attenuated radiation signal strength. In this experiment, the scanning parameters were set as: voltage $150 \mathrm{kV}$, current $180 \mu \mathrm{A}$, 400 projection images were obtained in one scan, and 1500 images were obtained after reconstruction. The image size was $2048 \times 2048$, and the resolution was $6.75 \mu$ $\mathrm{m}$.

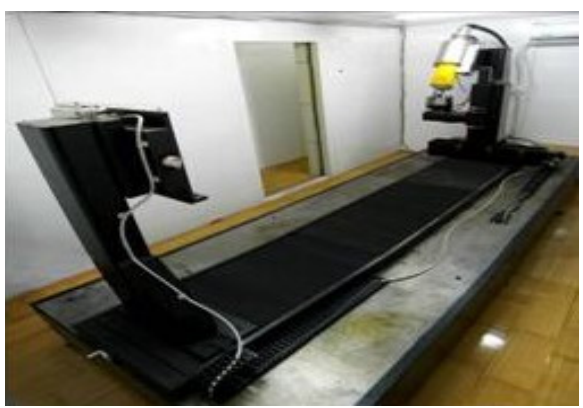

Fig.1. The micro-CT system

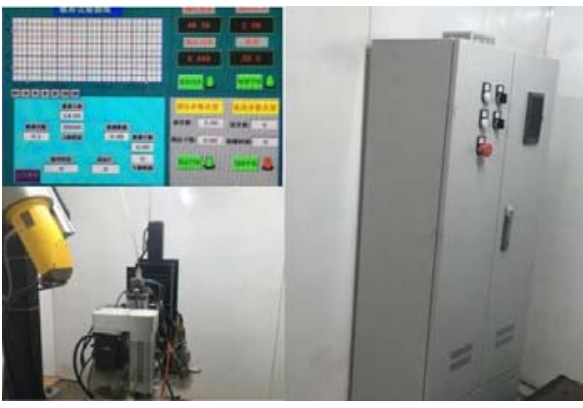

Fig. 2. THMC coupling test system

The THMC coupling test system can realize the test of rock cores under different coupling conditions. The specific experimental scheme of this test is: maintain constant confining pressure $1 \mathrm{MPa}$, and then apply axial compression with the pre-set loading speed, measure axial stress, strain and lateral deformation, and finally obtain the mechanical parameters of rock samples under triaxial condition. In some loading stages, the microscopic characteristics of crack propagation in the sample can be obtained by real-time CT scanning system. Figure 3 show the CT scanning slice image.

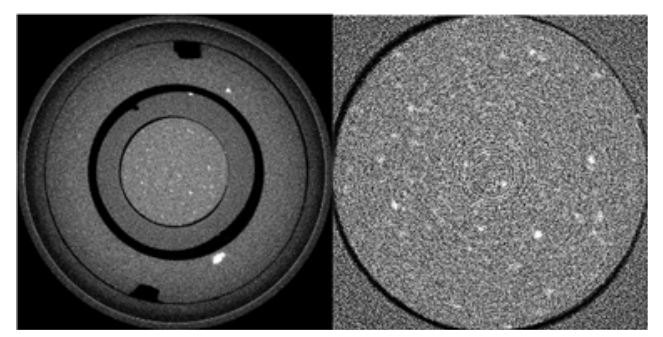

(a)

(b)

Fig. 3. (a)CT scan images of typical test sample. (b) Oil shales rock core sample 


\section{Results and discussion}

\subsection{Stress and strain characteristic of samples}

By applying load and deformation sensor to record the stress and strain of the sample, we can obtain the triaxial stress-strain curve of the oil shale sample at the confining pressure of $1 \mathrm{MPa}$, see Fig.4. Meanwhile, the oil shale samples were scanned at different loading stages by realtime CT to obtain the internal microscopic evolution characteristics. The curve in Fig. 4 shows that $\mathrm{S} 1$ sample exhibits obvious elastic deformation and plastic failure before peak stress. In the plastic failure stage, the sample has obvious characteristics of strain increase, and the plastic deformation is about twice as large as the elastic deformation. At the initial stage, the curve has a linear growth, and the sample is in the elastic deformation stage. With the load increasing, the plastic deformation of the sample accelerates, and the strain keeps grow until the sample is completely destroyed after the peak, and thus stress decreases. Since the test scheme was arranged to keep the load constant at one certain stage, the stress of the test sample would increase after reaching the peak, which had little influence on the experimental results. S2 sample also entered the linear elastic stage at the beginning. With the increase of the load, the slope of the curve basically remains unchanged and there is no obvious plastic deformation stage, so the yield point is difficult to identify. The elastic modulus of the two samples is basically the same, but the compressive strength of S1 is obviously higher than that of S2, which reflects the anisotropic characteristics of oil shale.

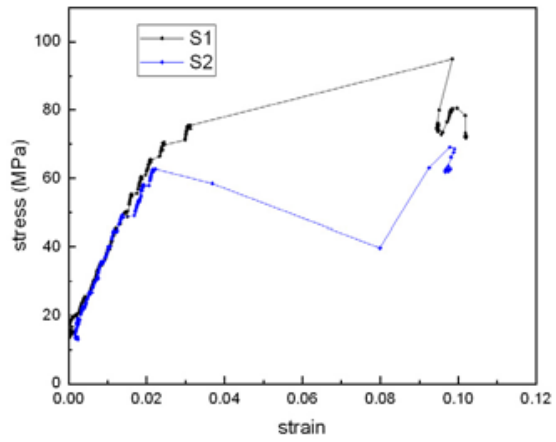

Fig.4. Stress-strain diagrams of sample S1 and sample S2

\subsection{The evolution characteristics of fractures}

Fig. 5 shows the slice images of oil shale samples under triaxial loading conditions, and further technical processing would carry out three-dimensional display and visualization of the image. The slice mage bit width is 8 bit, $0-255$ in gray range. The gray values of different rock type are different. The dark area in the figure is the void space, the bright part is the solid matrix, and the white part is the high-density mineral. CT images show that the oil shale is dense and heterogeneous in parallel bedding direction. The CT scan section shows that the internal structure of the oil shale sample is compact and the white high-density minerals distribute randomly and irregularly. Limited to the image resolution, the smaller size of changes cannot be observed. During the whole test, the lateral stress remained unchanged at $1 \mathrm{MPa}$, and the dynamic evolution law of the internal microstructure of oil shale samples was reproduced in real time during the continuous stage of axial stress. When the axial pressure $48.90 \mathrm{MPa}$ S1 specimen has not yet been observed visible cracks. When the stress reaches $57.79 \mathrm{MPa}$, cracks first appear in the direction of diameter, and the crack porosity is $1.00 \%$. When the stress increases to $62.23 \mathrm{MPa}$, the crack width grows obviously, and the crack porosity is $1.21 \%$. When the stress reached $66.68 \mathrm{MPa}$, the slice image showed that the specimen was completely destroyed, and the crack porosity reached $6.41 \%$. As for S2 specimen, when the axial pressure reached $57.79 \mathrm{MPa}$, cracks appeared in the direction of diameter for the first time, and the crack porosity is $1.21 \%$. And under the axial stress of $62.63 \mathrm{MPa}$, the specimen was completely destroyed, where the crack porosity reached $6.47 \%$.
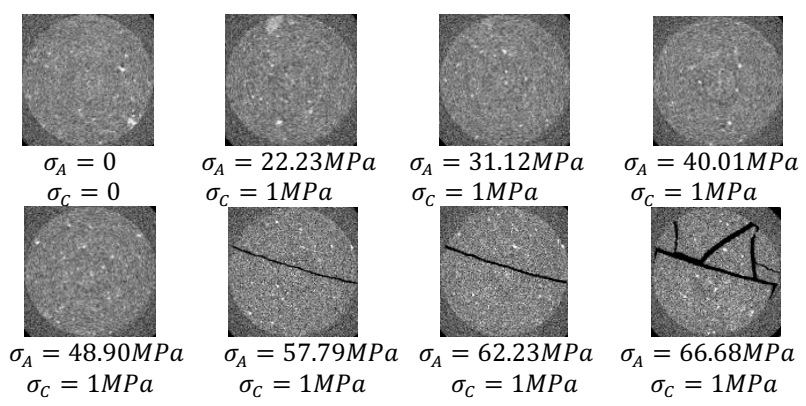

(a) Slices of S1 sample
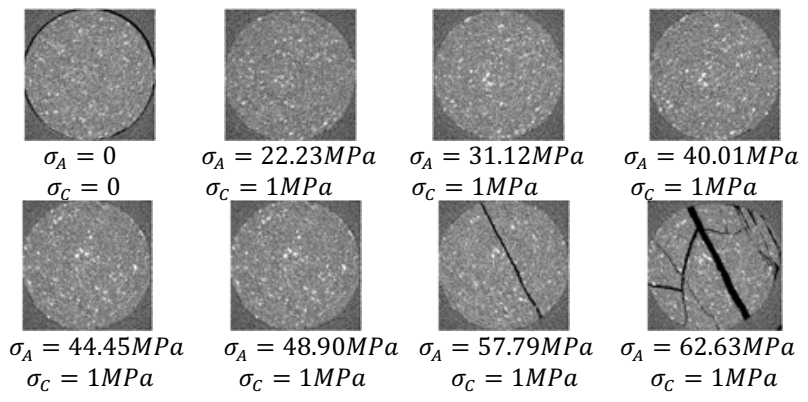

(b) Slices of S2 sample

Fig.5. Slices of oil shale samples

The CT-THMC coupling test system have given the internal microscopic evolution characteristic images of oil shale samples. In the previous section, S1 and S2 samples were qualitatively and quantitatively described under different loading conditions. The fracture evolution at different loading stages in the whole test is described in the following microscopic perspective.

Using VGStudio MAX software for multi-view display of CT scanning image. Under unload conditions, oil shale particles are dense and have no primary cracks, which can provide a reference for the evolution of internal microstructure at different loading stages, as can be seen in Fig.6. 


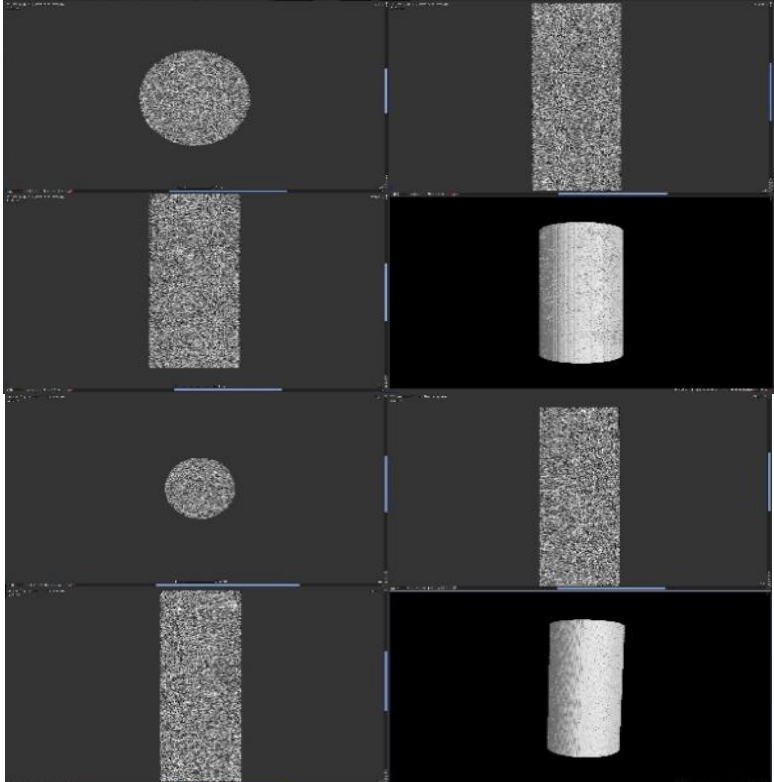

Fig.6. 3D view of specimen S1 and specimen S2 without loading

The initial crack of S1 specimen was parallel to the axis direction, which is in behaviour of tension rupture. When the stress continued to increase to $62.23 \mathrm{MPa}$, the main crack expanded along the axial direction and its opening increased apparently. When the stress reached $66.68 \mathrm{MPa}$, the specimen was completely destroyed and the shear crack with an angle to the axial direction appears, see Fig.7. The specimen S2 initially captured the first crack at the stress of $57.79 \mathrm{MPa}$, which was a parallel axial tensile crack. Under the action of continuously increasing stress, multiple cracks were generated in the S2 specimen, and are connected and overlapped with each other to form a connected network. At this time, the strength reached the peak stress and was completely damaged. The failure form is combined with tensile failure and shear failure, see Fig.8.

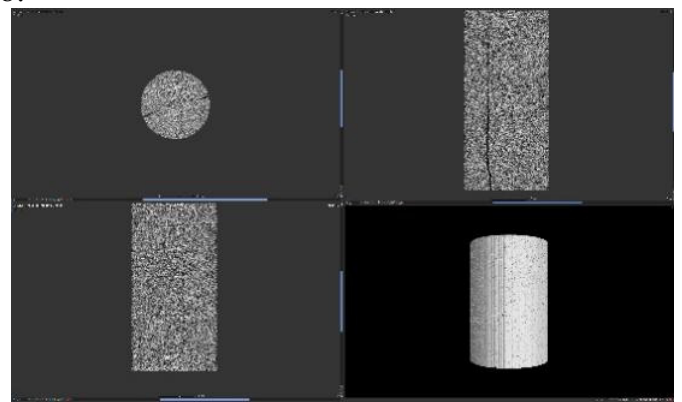

(a)

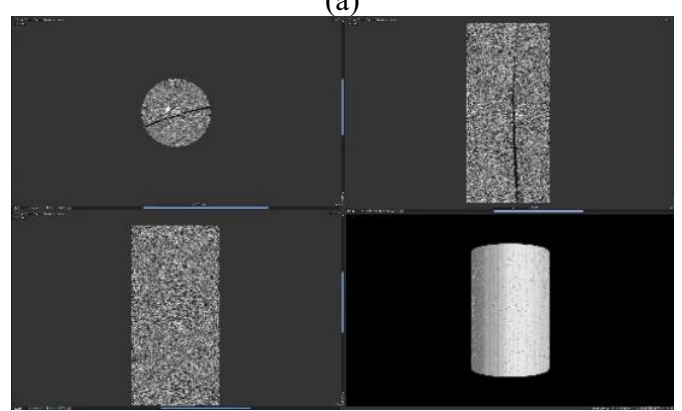

(b)

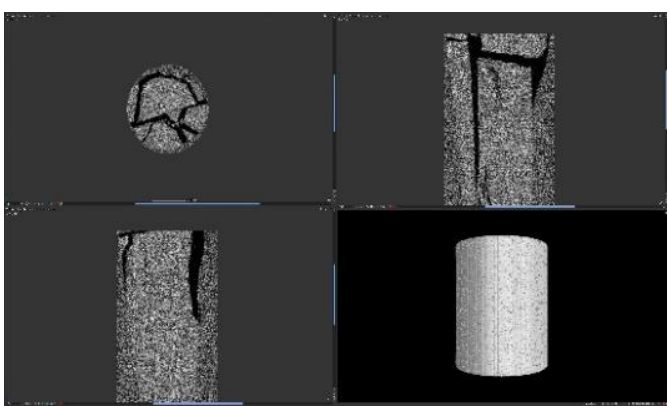

(c)

Fig.7. Fracture evolution of S1 specimen

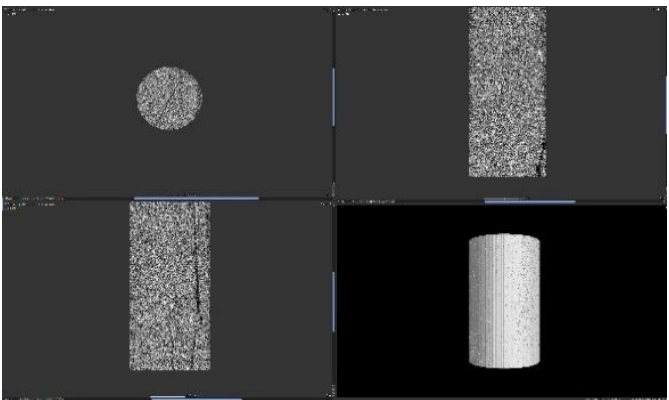

(a)

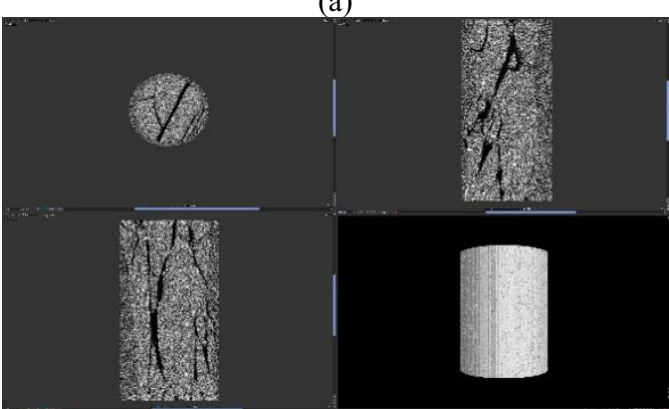

(b)

Fig.8. Fracture evolution of S2 specimen

\section{Conclusion}

In this paper, the triaxial mechanical properties and realtime meso-structural evolution characteristics of oil shale were studied by CT-THMC coupling test system, and the following conclusions were drawn:

(1) CT scan results showed that the oil shale structure is dense and the internal material distribution is irregular. Under triaxial stress conditions, the compressive strength display obvious anisotropy, but the elastic modulus is basically the same in different bedding directions.

(2) CT images showed that initial cracks were observed in S1 and S2 specimens at 57.79MPa under load process. With the increase of stress, the fracture continues to expand and the fracture damage continues to accumulate, indicating that the stress-strain curve is in the irreversible stage of deformation.

(3) With the increase of triaxial stress, the microcracks of oil shale samples expand simultaneously in axial and lateral directions, and the initial stage is mainly tensile failure. As the stress continues to increase, there will be shear failure in the interior of oil shale sample. Eventually, the specimen was completely destroyed under the joint action. 


\section{Acknowledgements}

This work was funded by the National Natural Science Foundation of China (11772213).

\section{References}

1. Y. Zhang, G. Feng, M. Zhang, H. Ren, J. Bai, Y. Guo, H. Jiang, J. Kang, Energy Policy 96 (2016)

2. J. Wang, L. Feng, M. Steve, X. Tang, T.E. Gail, H. Mikael, Energy 82 (2015)

3. P.R. Tisot, J. Chem. Eng. Data 12, 3 (1967)

4. F.E.W. Duvall, H.Y. Sohn, C.H. Pitt, Fuel 64, 2 (2015)

5. A. K. Burnham, Fuel 219 (2018)

6. Joshua A. White, A. K. Burnham, David W. Camp, Rock Mech. Rock Eng. 50 (2017)

7. Z. Kang, W. Wang, W. Cao, W. Liang, Z. Chang, Z. Liu, J. Taiyuan University of Technology 6 (2013)

8. G. Wang, D. Yang, Y. Zhao, Z. Kang, J. Zhao, X. Huang, Appl. Therm. Eng. 146 (2018)

9. D. Yang, G. Wang, Z. Kang, J. Zhao, Y. Lv, Nat. Resour. Res. 29, 1 (2020)

10. D. Yang, Y. Zhao, Z. Kang, Oil Shale 36, 4 (2019)

11. Y. Fan, L. Durlofsky, H.A. Tchelepi, Spe. J. 15, 2 (2009)

12. K.J. Lee, G.J. Moridis, C.A. Ehlig-Economides, Energ. Explor. Exploit. 35, 3 (2017)

13. Y. Ju, C. Xi, Y. Zhang, L. Mao, F. Gao, H. Xie, Rock Mech. Rock Eng. 51 (2018)

14. Y. Zhang, M. Lebedev, Y. Jing, H. Yu, S. Iglauer, Int. J. Coal Geol. 203 (2019)

15. T. Saif, Q. Lin, Y. Gao, Y. Al-Khulaifi, F. Marone, D. Hollis, M.J. Blunt, B. Bijeljic, Appl. Energ. 235 (2019) 